\title{
SP600125 suppresses Keap1 expression and results in NRF2-mediated prevention of diabetic nephropathy
}

\author{
Huan Zhang1, Xiuxia Liu², Shanshan Zhou33, Ye Jia4, Ying Lij, Yuguo Song6,7, Junnan Wang ${ }^{8}$ and Hao Wu9,10 \\ 'Operating Theater, China-Japan Union Hospital of Jilin University, Changchun, Jilin, People's Republic of China \\ 2Department of Clinical Laboratory, The Second Hospital of Jilin University, Changchun, Jilin, People's Republic of China \\ ${ }^{3}$ Cardiovascular Center, The First Hospital of Jilin University, Changchun, Jilin, People's Republic of China \\ ${ }^{4}$ Department of Nephrology, The First Hospital of Jilin University, Changchun, Jilin, People's Republic of China \\ ${ }^{5}$ Department of Dermatology, Affiliated Hospital of Beihua University, Jilin, Jilin, People's Republic of China \\ ${ }^{6}$ Research Institute of Clinical Immunology, Affiliated Hospital of Beihua University, Jilin, Jilin, People's Republic of China \\ ${ }^{7}$ Research Center for Life Sciences, Beihua University, Jilin, Jilin, People's Republic of China \\ ${ }^{8}$ Department of Cardiology, The Second Hospital of Jilin University, Changchun, Jilin, People's Republic of China \\ ${ }^{9}$ Department of Nephrology, The Second Hospital of Jilin University, Changchun, Jilin, People's Republic of China \\ 10The '973' National Basic Research Program of China, Changchun University of Chinese Medicine, Changchun, Jilin, People's Republic of China
}

Correspondence should be addressed to H Wu: wuhaobaha@jlu.edu.cn or to J Wang: jdeywjn@163.com

\begin{abstract}
c-Jun N-terminal kinase (JNK) contributes to the pathogenesis of diabetic nephropathy (DN). The JNK inhibitor SP600125 was reported to ameliorate DN. However, the mechanism remained unclear. We previously reported that SP600125 activated nuclear factor erythroid 2-related factor 2 (NRF2), a governor of the cellular antioxidant defense system, in the aortas of the diabetic mice. Given the critical role of NRF2 in preventing DN, the present study aimed to test whether or not NRF2 is required for SP600125's protection against DN. To test the role of NRF2 in SP600125's effect, streptozotocininduced C57BL/6 wild-type (WT) and Nrf2-knockout (KO) diabetic mice were treated in the presence or absence of SP600125, for 24 weeks. To explore the mechanism by which SP600125 activates NRF2, mouse mesangial cells (MMCs) were treated with high glucose (HG), in the presence or absence of either SP600125 or JNK siRNA. SP600125 significantly attenuated the diabetes-induced renal oxidative stress, inflammation, fibrosis, pathological change and dysfunction in the WT, but not the Nrf2 KO mice. SP600125 inactivated JNK, inhibited kelch-like ECH-associated protein 1 expression, preserved NRF2 protein and facilitated its nuclear translocation in the kidneys of the WT mice, the effects of which were similarly produced by either SP600125 or JNK siRNA in HG-treated MMCs. Further, both SP600125 and JNK siRNA alleviated HG-induced mesangial oxidative stress and expression of inflammatory and fibrotic genes. The present study demonstrates that NRF2 is required for SP600125's protection against DN. SP600125 activates NRF2 possibly via inhibition of JNK-induced Keap1 expression.
\end{abstract}

Key Words
- antioxidant
- diabetic nephropathy
- KEAP1
- JNK
- NRF2

Journal of Molecular Endocrinology (2018) 60, 145-157 


\section{Introduction}

Diabetic nephropathy (DN) is the main cause of endstage renal disease (Dronavalli et al. 2008), and to date, has not been effectively treated. Therefore, it is essential to discover novel targets and develop more effective medicines to prevent the development of DN.

c-Jun N-terminal kinase (JNK) belongs to the mitogenactivated protein kinases (MAPK) family and regulates the transcription activity of activator protein 1 (AP-1) by phosphorylation of c-Jun (Davis 2000). AP-1 activates the transcription of a cohort of genes, such as transforming growth factor beta 1 (Tgf- $\beta 1$ ) (Weigert et al. 2000) and fibronectin (Fn) (Lan et al. 2013), which play important roles in the initiation and progression of DN (Schena \& Gesualdo 2005). Enhanced JNK activity was observed in high glucose (HG)-treated mesangial cells (Zhou et al. 2014, Gao et al. 2016), in kidneys of diabetic mice (Pan et al. 2013, Wang et al. 2015, Gao et al. 2016) and in kidney biopsy tissue from patients with DN (De Borst et al. 2007). Therefore, targeting JNK could be a promising strategy in prevention of DN. SP600125 is a specific inhibitor of JNK (Bogoyevitch et al. 2004). Previous studies reported the beneficial effect of SP600125 on DN (Wang et al. 2015, Gao et al. 2016, Hong et al. 2016). Despite the finding of decreased expression of several pro-fibrotic and proinflammatory genes, the mechanism through which SP600125 ameliorated DN was unclear.

Nuclear factor erythroid 2-related factor 2 (NRF2) is a master regulator of cellular antioxidant defensive activity (Zheng et al. 2011). As a transcription factor, NRF2 turns on the transcription of various antioxidant genes, such as heme oxygenase 1 (Ho1) and $\mathrm{NAD}(\mathrm{P}) \mathrm{H}$ dehydrogenase (quinone) 1 (Nqo1), leading to the increased antioxidants which function as scavengers for diabetes-induced free radicals (Ruiz et al. 2013). Accumulating evidence has demonstrated the pivotal role of NRF2 in self-defense against the pathogenesis of DN, since the Nrf2 geneknockout (KO) mice developed more severe nephropathy under diabetic condition, as compared with the wild-type (WT) mice (Jiang et al. 2010, Wu et al. 2015, 2016). In our previous study, SP600125 was found to activate NRF2 in the aortas of the diabetic mice (Liu et al. 2014), suggesting that JNK may negatively regulate NRF2. However, whether or to what extent NRF2 is required for SP600125's protective effect was unknown. By using Nrf2 KO mice, the present study aimed to explore the role of NRF2 in SP600125's protection against DN.

Kelch-like ECH-associated protein 1 (KEAP1) is a key negative regulator of NRF2 (Kensler et al. 2007). KEAP1 sequesters NRF2 in cytoplasm, restricting NRF2 from nuclear translocation and promoting its proteasomal degradation (Zhang \& Hannink 2003). JNK might be an upstream regulator of KEAP1, since 36 c-Jun-binding sites were found between $-3000 \mathrm{bp}$ and $-1 \mathrm{bp}$ within the promoter region of the mouse Keap1 gene (Jaspar Database). Thus, JNK might inhibit NRF2 through induction of Keap1 expression. By determining the renal level of Nrf2 mRNA, the preservation of NRF2 protein, the proportion of the NRF2 nuclear translocation and the expression of Keap1, the present study tested the actions of SP600125 in the regulation of NRF2. To further verify the effect of JNK inhibition on NRF2 signaling and HG-induced injury, SP600125 was studied in HG-treated mouse mesangial cells (MMCs), by comparing to the effect of JNK siRNA.

In summary, the present study aimed to answer the following questions: (1) whether or not SP600125 activates NRF2 in DN; (2) whether or to what extent NRF2 is required for SP600125's protection against DN and (3) how does SP600125 activate NRF2.

\section{Materials and methods}

\section{Animal treatment}

C57BL/6 WT (Nrf2+/+) and Nrf2 KO (Nrf2-/-) mice were obtained through breeding of heterozygotes (Nrf2+/-) (Zheng et al. 2011, Dong et al. 2017). All mice were housed in the Animal Center of Jilin University at $22^{\circ} \mathrm{C}$, on a 12:12-h light-darkness cycle with free access to rodent feed and tap water. The Institutional Animal Care and Use Committee at Jilin University approved all experimental procedures for these animals.

Eight-week-old male mice received either sodium citrate or streptozotocin (STZ, $50 \mathrm{mg} / \mathrm{kg}$ daily, dissolved in $0.1 \mathrm{M}$ sodium citrate, $\mathrm{pH} 4.5$; Sigma-Aldrich) through intraperitoneal injection for 5 consecutive days. One week after the last injection of STZ, fasting glucose levels (4-h fast) were measured. Mice with a fasting glucose level above $13.89 \mathrm{mM}$ were considered diabetic. Diabetic and age-matched control mice were then given by gavage with SP600125 $(5 \mathrm{mg} / \mathrm{kg}$, dissolved in 1\% CMC-Na solution; Medchem Express, Shanghai, PRC) or 1\% CMC-Na solution, once every 2 days (Pan et al. 2014), for 24 weeks.

Blood glucose was recorded on days $0,28,56,84,112$, 140 and 168 post diabetes onset. Urinary albumin and creatinine were recorded on day 168 post diabetes onset. The mice were then killed and their kidneys harvested for analysis. 


\section{Analysis of kidney dysfunction}

A mouse albumin kit (Bethyl Laboratories, Montgomery, TX, USA) and a QuantiChrom Creatinine Assay Kit (BioAssay Systems, Hayward, CA, USA) were used to determine urinary albumin and creatinine on spot urine samples following the manufacturer's instructions. Urinary albumin-to-creatinine ratio (UACR) was calculated to reflect kidney dysfunction.

\section{Morphological analysis}

After harvesting, the kidney tissues were fixed immediately in $10 \%$ buffered formalin solution and were embedded in paraffin and sectioned into 5- $\mu \mathrm{m}$-thick sections onto glass slides. The sections were processed for Periodic acid-Schiff (PAS) and Masson's trichrome staining. Morphometric analysis was quantified using Image-Pro Plus 6.0 software (Media Cybernetics, Bethesda, MD, USA). Selection of areas to photograph and scoring was done by people blind to the identity of the samples.

\section{Immunohistochemical (IHC) analysis}

IHC staining was performed as previously described ( $\mathrm{Wu}$ et al. 2014) to assess the status of renal oxidative damage and antioxidant activity, using primary antibodies against 3-nitrotyrosine (3-NT, Millipore; 1:100), HO1 (Santa Cruz Biotechnology, 1:100) and NQO1 (Santa Cruz Biotechnology, 1:100).

\section{Cell culture and experiments}

MMCs were isolated and cultured as previously described (Sun et al. 2017). Passages 5-7 were used for the experiments (Sun et al. 2017). To determine the effect of SP600125 and JNK siRNA on JNK activity, Keap1 expression, NRF2 signaling and HG-induced injury, MMCs were subjected to $\mathrm{HG}(25 \mathrm{mM})$, in the presence or absence of $10 \mu \mathrm{M}$ SP600125 (Ho et al. 2007, Zhou et al. 2014, Gao et al. 2016) or JNK siRNA (100nM, GenePharma, Suzhou, Jiangsu, PRC), for $48 \mathrm{~h}$. The negative control siRNA was purchased from GenePharma. The transfection reagent RFect was provided by Changzhou Bio-generating Biotechnologies, Changzhou, PRC.

\section{Western blot analysis}

Western blot analysis was performed using kidney cortex as previously described (Cai et al. 2005). The primary antibodies were anti-connective tissue growth factor (CTGF, Santa Cruz Biotechnology, 1:500), anti-GAPDH (Santa Cruz Biotechnology, 1:3000), anti-histone H3 (Santa Cruz Biotechnology, 1:1000), anti-HO1 (Santa Cruz Biotechnology, 1:1000), anti-induciblenitricoxidesynthase (iNOS, Cell Signaling Technology, 1:1000), anti-total cellular c-Jun (t-c-Jun, Cell Signaling Technology, 1:500), anti-total cellular JNK (t-JNK, Cell Signaling Technology, 1:1000), anti-KEAP1 (Santa Cruz Biotechnology, 1:500), anti-NQO1 (Santa Cruz Biotechnology, 1:1000), anti-NRF2 (Santa Cruz Biotechnology, 1:1000), anti-phosphorylatedc-Jun (p-c-Jun, Cell Signaling Technology, 1:500), antiphosphorylated-JNK (p-JNK, Cell Signaling Technology, 1:500), anti-TGF- $\beta 1$ (Cell Signaling Technology, 1:1000), anti-tumor necrosis factor alpha (TNF- $\alpha$, Cell Signaling Technology, 1:500) and anti-vascular cell adhesion molecule 1 (VCAM-1, Santa Cruz Biotechnology, 1:1000).

\section{Real-time PCR}

Quantitative real-time PCR was performed as previously described (Wang et al. 2009, Wu et al. 2014). Primers for collagen 4 (Col4), Fn, Gapdh, Nqo1, intercellular adhesion molecule 1 (Icam-1), Ho1, iNos, Keap1, Nrf2 and Vcam-1 were all purchased from Life Technologies.

\section{Quantitative analysis of lipid peroxides}

Renal malondialdehyde (MDA) concentration was calculated following the instructions by a lipid peroxidation assay kit from Nanjing Jiancheng Bioengineering Institute, Nanjing, Jiangsu, PRC.

\section{Isolation of nuclei}

Nuclei of renal tissue and MMCs were isolated using a nuclei isolation kit (Sigma-Aldrich) as previously described (Dong et al. 2017, Sun et al. 2017), following the manufacturer's instructions.

\section{Statistical analysis}

Eight mice per group were studied. The measurements for each group were summarized as means \pm s.D. Image Quant 5.2 (GE Healthcare Bio-Sciences) was used to analyze Western blots. Two-way ANOVA was performed for the comparisons among different groups of the two types of the mice. One-way ANOVA was performed for comparisons among different groups of either the WT or the Nrf2 KO mice, as well as different groups of the cell 
experiments. Post hoc pairwise comparisons were then performed using Tukey's test with Origin 8.6 data analysis and graphing software Lab (OriginLab, Northampton, MA, USA). A test is significant if $P<0.05$.

\section{Results}

\section{Deletion of the Nrf2 gene led to a complete abolishment of SP600125's protection against diabetes-induced albuminuria and renal pathological change}

Blood glucose levels were monitored every four weeks post diabetes onset. Both the WT and the Nrf2 KO diabetic mice had significantly increased blood glucose levels at all the time points, as compared with their respective controls (Fig. 1A). SP600125 did not alter blood glucose under both the diabetic and the non-diabetic conditions, in both types of the mice (Fig. 1A). No significant difference in blood glucose level was detected between the WT and the Nrf2 KO mice, under both the diabetic and non-diabetic conditions (Fig. 1A). However, as compared with the WT diabetic mice, the Nrf2 KO diabetic mice developed remarkably higher UACR (Fig. 1B) and more severe renal pathological change, as shown by PAS staining (Fig. 1C) and Masson's trichrome staining (Fig. 1D), including the more drastic increase in glomerular area (Fig. 1E), mesangial matrix (Fig. 1F) and Masson's positive area (Fig. 1G). These results confirmed the critical role of NRF2 in self-defense against DN. Notably, in the WT mice, SP600125 significantly attenuated the diabetes-induced elevation of UACR (Fig. 1B, left panel) and pathological change (Fig. 1E, F and G, left panels). However, these beneficial effects of SP600125 did not exist in the Nrf2 KO mice (Fig. 1B, E and F, right panels), with an exception of the amelioration of renal fibrosis (Fig. 1G, right panel). Nonetheless, the efficacy of SP600125 in decreasing renal fibrosis was blunted in the absence of NRF2, since SP600125 decreased renal fibrosis by $58.10 \%$ in the WT mice, whereas only by $25.47 \%$ in the $N r f 2 \mathrm{KO}$ mice (Fig. 1G). Therefore, NRF2 plays a major role in SP600125's protection against the diabetes-induced albuminuria and renal pathological change.

\section{NRF2 was required for SP600125's alleviation of the diabetes-induced renal oxidative stress, inflammation and fibrosis}

Renal nitrosative damage was reflected by IHC staining of 3-NT (Fig. 2A), which showed a preferable expression of 3-NT in the glomeruli. Further, renal oxidative stress, inflammation and fibrosis were evaluated at the molecular level. The levels of the oxidative stress indicators MDA (Fig. 2B) and iNOS protein (Fig. 2C), the protein levels of the pro-inflammatory markers TNF- $\alpha$ (Fig. 2D) and VCAM-1 (Fig. 2E), and the protein levels of the profibrotic parameters TGF- $\beta 1$ (Fig. 2F) and CTGF (Fig. 2G) were measured in both types of the mice, all of which were elevated under the diabetic condition (Fig. 2B, C, D, E, F and G). SP600125 attenuated these indices in the WT, but not the Nrf2 KO, diabetic mice (Fig. 2B, C, D, E and F), except for a mild decrease $(26.74 \%)$ in CTGF in the Nrf2 KO diabetic mice (Fig. 2G, right panel), in contrast to the WT mice (47.97\%, Fig. 2G, left panel). These results indicate that NRF2 predominantly mediates SP600125's protection against diabetes-induced renal oxidative stress, inflammation and fibrosis.

\section{SP600125 preserved renal NRF2 protein and facilitated NRF2 nuclear translocation and function, without altering Nrf2 mRNA}

Renal Nrf2 expression and function were evaluated in the following study. Neither Nrf2 mRNA (Fig. 3A, right panel) nor NRF2 protein (Fig. 3B, right panel) were detectable in the kidneys of Nrf2 $\mathrm{KO}$ mice, as a confirmation of the Nrf2 gene deletion. SP600125 did not alter Nrf2 mRNA in the WT mice (Fig. 3A, left panel). Rather, it increased total cellular NRF2 (t-NRF2, Fig. 3B, left panel) and nuclear NRF2 (n-NRF2, Fig. 3C, left panel) in both the WT diabetic and non-diabetic mice. These results suggest that SP600125 regulates Nrf2 expression at the protein, but not the mRNA level. NRF2 nuclear translocation was further evaluated by calculating the ratio of n-NRF2/histone $\mathrm{H} 3$ to t-NRF2/GAPDH, which was enhanced by SP600125 under both the diabetic and the non-diabetic conditions (Fig. 3D). Further, the mRNA levels of $\mathrm{Ho} 1$ and Nqo1 were both significantly increased by SP600125 in the WT, but not Nrf2 KO mice (Fig. 3E and F). It is noted that, compared to the WT mice, the Nrf2 KO mice expressed less renal Ho1 and Nqo1 mRNAs under both the diabetic and nondiabetic conditions (Fig. 3E and F), a result in line with the renal susceptibility of the Nrf2 $\mathrm{KO}$ mice upon diabetes (Fig. 1B, C, D, E, F and G). Renal expression of HO1 and NQO1 was further assessed by IHC staining, which showed an increase of these antioxidants by SP600125 in the glomeruli of the WT mice (Fig. 3G and H, upper panels). This effect did not exist in the glomeruli of the Nrf2 KO mice (Fig. 3G and H, lower panels). Collectively, SP600125 was found to activate NRF2 signaling via preserving NRF2 
A

$\begin{array}{ll}\triangle \text { WT Ctrl } & \triangle \text { KO Ctrl } \\ \text {-WT Ctrl/JNKi } & \nabla \text { KOCtrl/JNKi } \\ \text { WT DM } & \triangleleft \text { KODM } \\ \text {-WT DM/JNKi } & \triangleright \text { KODM/JNKi }\end{array}$

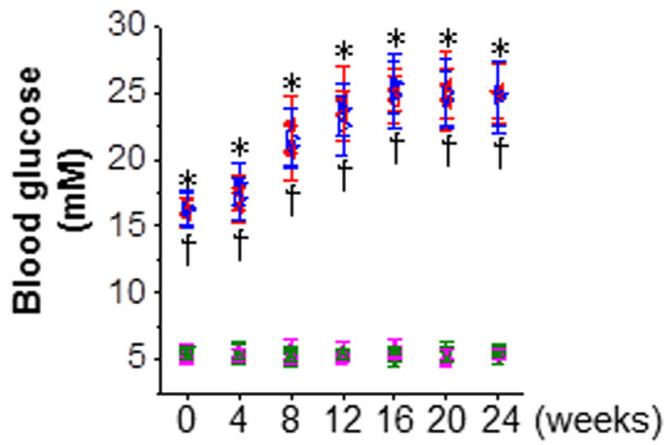

C

PAS staining

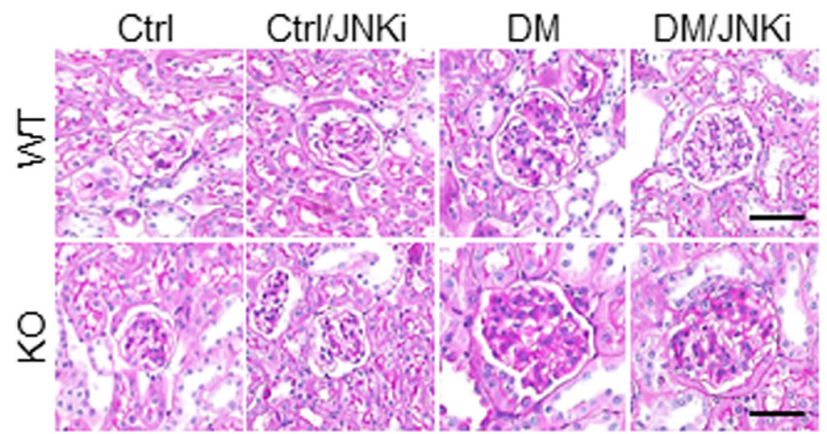

B
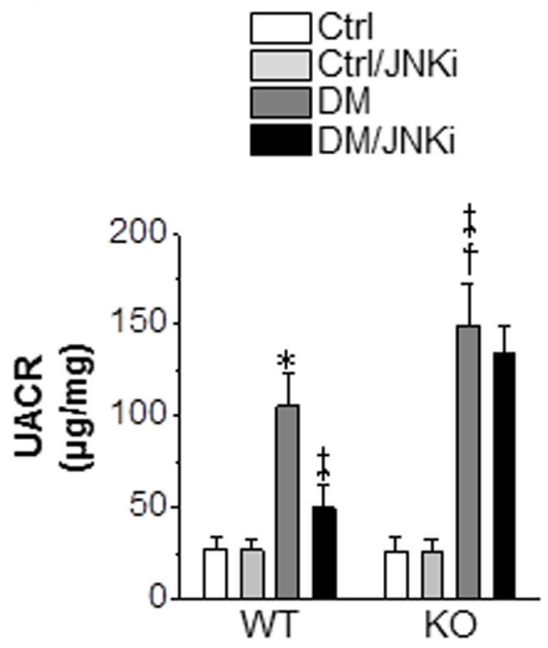

D

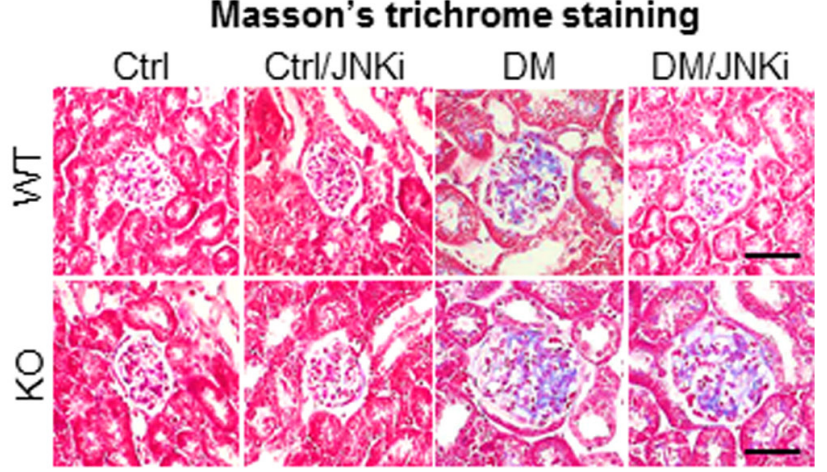

G

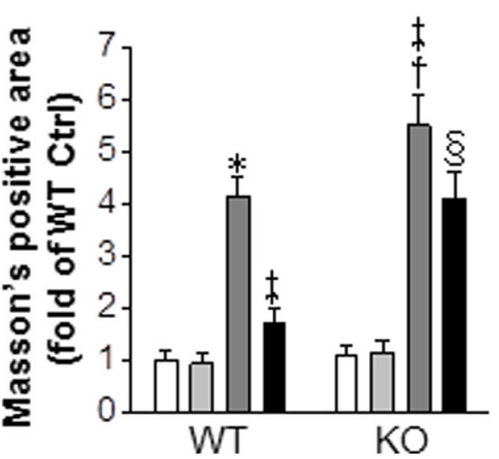

Figure 1

Deletion of the Nrf2 gene led to a complete abolishment of SP600125's protection against diabetes-induced albuminuria and renal pathological change. Eight-week-old C57BL/6 WT and Nrf2 KO male mice were induced to diabetes by streptozotocin. (A) Blood glucose levels were determined every four weeks post diabetes onset. Urinary albumin and creatinine were recorded at 24 weeks post diabetes onset with (B) UACR calculated. To evaluate renal pathological change, (C) PAS and (D) Masson's trichrome staining were performed, with (E) glomerular area and (F) mesangial matrix expansion quantified from PAS staining and (G) Masson's positive area quantified from Masson's trichrome staining. For ( $E$, $F$ and $G$ ), the data are normalized to WT Ctrl. All the data are presented as means \pm s.D. $(n=8) .{ }^{*} P<0.05$ vs WT Ctrl; ${ }^{\dagger} P<0.05$ vs KO Ctrl; ${ }^{\ddagger} P<0.05$ vs WT DM; ${ }^{\S} P<0.05$ vs KO DM. Bar $=50 \mu \mathrm{m}$. Scatter plots: solid up triangle, WT Ctrl; solid down triangle, WT Ctrl/JNKi; solid left triangle, WT DM; solid right triangle, WT DM/JNKi; hollow up triangle, KO Ctrl; hollow down triangle, KO Ctrl/JNKi; hollow left triangle, KO DM; hollow right triangle, KO DM/JNKi. Bars: white, Ctrl; light gray, Ctrl/JNKi; dark gray, DM; black, DM/JNKi. Ctrl, control; DM, diabetes mellitus; JNKi, the JNK inhibitor SP600125; KO, knockout; PAS, Periodic acid-Schiff; UACR, urinary albumin to creatinine ratio; WT, wild type. 
A
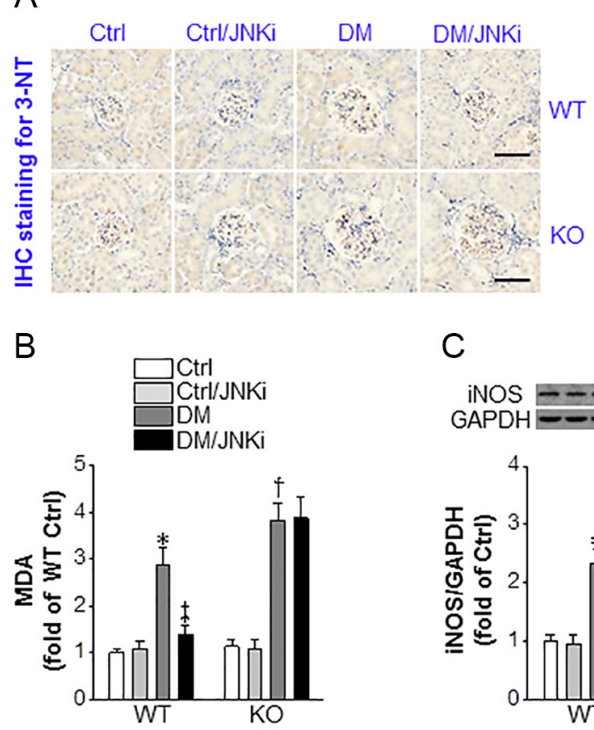

C
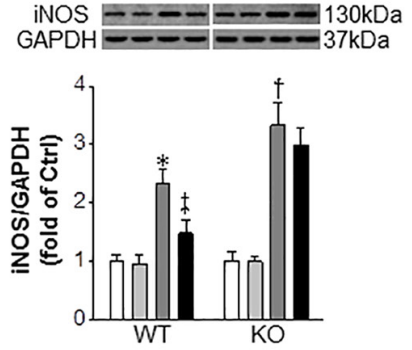

D

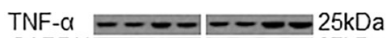

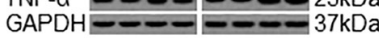

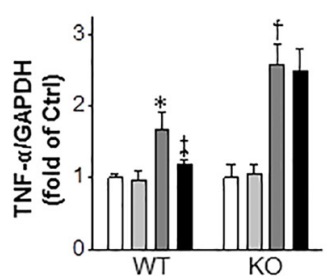

$\mathrm{F}$
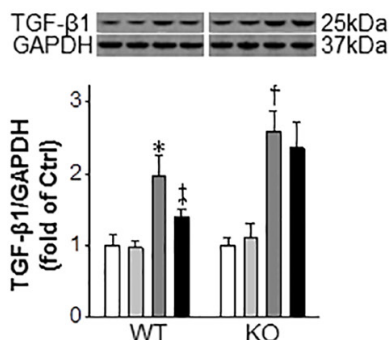

E

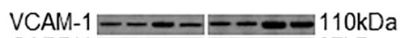
$\mathrm{GAPDH}=-37 \mathrm{kDa}$

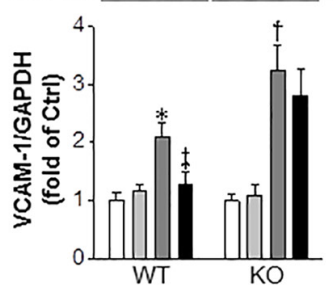

G
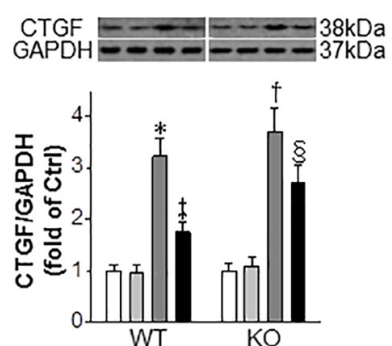

Figure 2

NRF2 was required for SP600125's alleviation of the diabetes-induced renal oxidative stress, inflammation and fibrosis. To further test the role of NRF2 in SP600125's protection against diabetes-induced renal injury, $\mathrm{IHC}$ staining was performed to assess renal expression of (A) 3-NT, an indicator of nitrosative damage. Further, renal (B) MDA levels were measured by an MDA assay kit and protein levels of (C) iNOS, (D) TNF- $\alpha$, (E) VCAM-1, (F) TGF- $\beta 1$ and (G) CTGF were determined by Western blot. For (B), the data is normalized to WT Ctrl. For (C, D, E, F and G), the date is normalized to either WT Ctrl or KO Ctrl, respectively. All the data are presented as means \pm s.D. $(n=8) .{ }^{*} P<0.05$ vs WT $C$ trl; ${ }^{+} P<0.05$ vs KO $C$ trl; ${ }^{\ddagger} P<0.05$ vs WT DM; $\$ P<0.05$ vs KO DM. Bars: white, Ctrl; light gray, Ctrl/ JNKi; dark gray, DM; black, DM/JNKi. 3-NT, 3-nitrotyrosine; CTGF, connective tissue growth factor; IHC, inmmunohistochemical; iNOS, inducible nitric oxide synthase; MDA, malondialdehyde; TGF- $\beta 1$ transforming growth factor beta 1 ; TNF- $\alpha$, tumor necrosis factor alpha; VCAM-1, vascular cell adhesion molecule 1. Other abbreviations are the same as those in Fig. 1.

protein and facilitating its nuclear translocation, rather than enhancing Nrf2 transcription.

\section{SP600125 decreased Keap1 expression through inhibition of JNK activity}

Given that KEAP1 restricts NRF2 from nuclear translocation and promotes proteasomal degradation of NRF2 (Zhang \& Hannink 2003), we speculated that the observed preservation and relocation of NRF2 by SP600125 might be due to a downregulated KEAP1 expression or function. To test this hypothesis, renal Keap1 expression was determined. As expected, SP600125 markedly decreased Keap1 mRNA (Fig. 4A) and protein (Fig. 4B) in both types of the mice, under both the diabetic and the non-diabetic conditions. The inhibitory effect of SP600125 on JNK activity was further verified by the decreased phosphorylation of renal JNK (Fig. 4C) and c-Jun (Fig. 4D).

The following study was to further test whether inhibition of JNK activity could lead to a downregulated Keap1 expression. Thus, HG-treated MMCs were subjected to either SP600125 or JNK siRNA. MMC was selected for the study because of the diabetes-induced remarkable pathological change observed in Fig. 1C, D, E, F and G. SP600125 decreased the ratio of p-JNK to t-JNK (Fig. 4E, left panel), but not t-JNK to GAPDH (Fig. 4E, middle panel). On the contrary, the ratio of t-JNK to GAPDH, but not p-JNK to t-JNK, was drastically decreased by JNK siRNA (Fig. 4E, left and middle panels). Despite these distinct actions of SP600125 and JNK siRNA (Fig. 4E, left and middle panels), both of them led to a significant decrease in the ratio of p-JNK to GAPDH (Fig. 4E, right panel). Inactivation of JNK by SP600125 and JNK siRNA was further confirmed by the decreased phosphorylation of c-Jun (Fig. 4F). SP600125 and JNK siRNA also led to an inhibition of Keap1 expression at both the mRNA (Fig. 4G) and the protein (Fig. 4H) levels, the result of which was in accordance with the findings in vivo (Fig. $4 \mathrm{~A}$ and B).

Taken together, by comparing with the effect of JNK siRNA, SP600125 was found to inhibit Keap1 expression via inactivation of JNK.

\section{Both SP600125 and JNK siRNA preserved NRF2 protein and enhanced its nuclear translocation and function}

Given the verified JNK inactivation-induced inhibition of Keap1 expression (Fig. 4G and H), the following study 
A
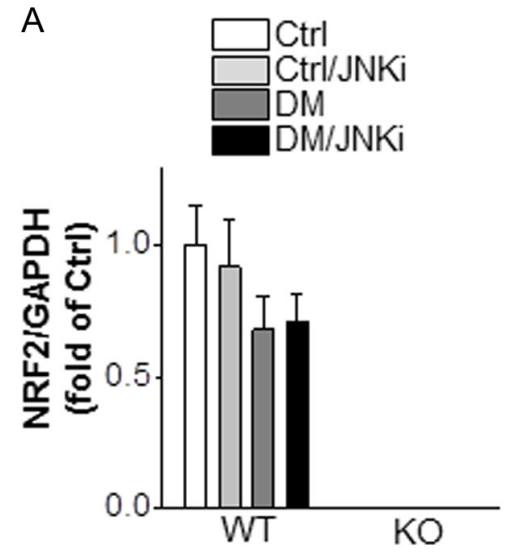

D

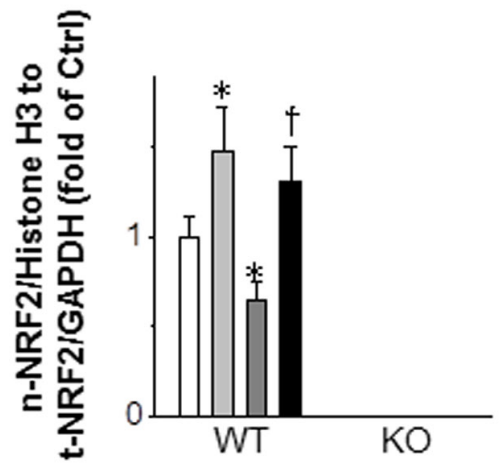

B
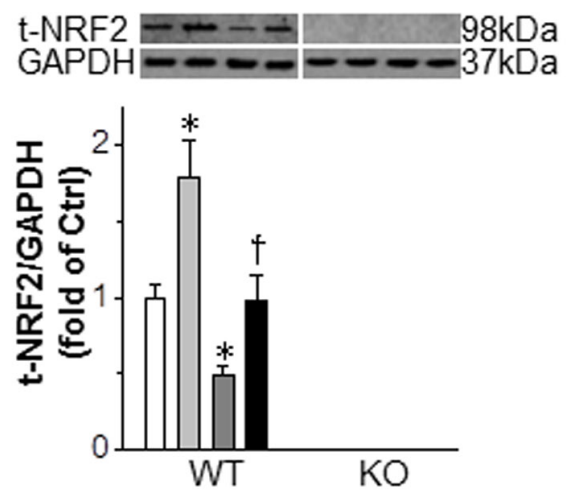

E

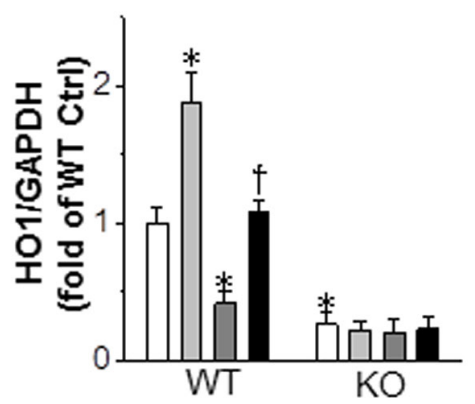

C
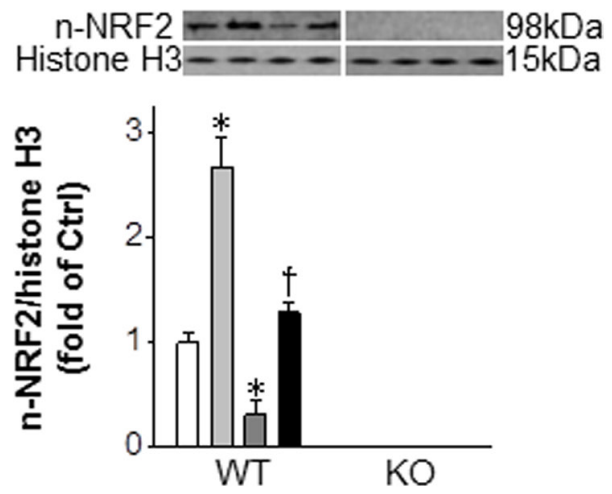

F
G

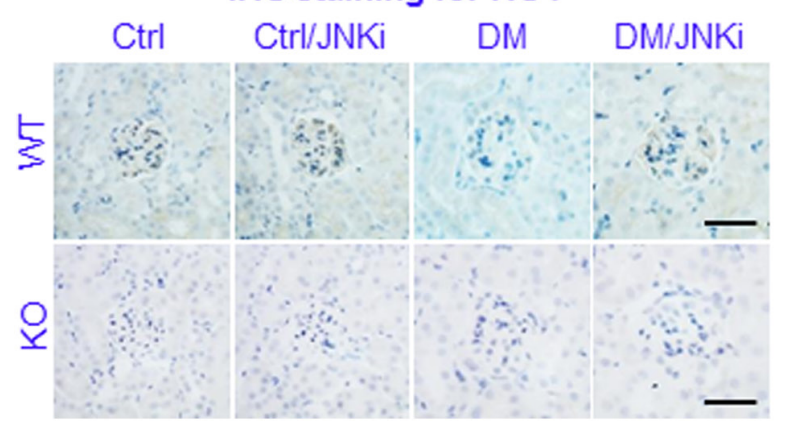

$\mathrm{H}$

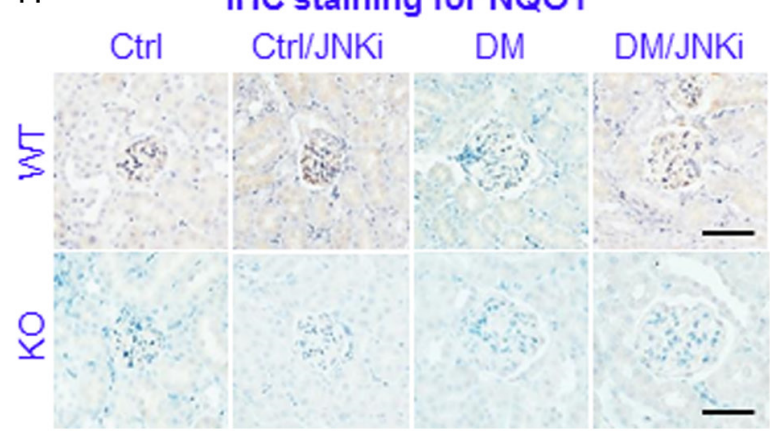

Figure 3

SP600125 preserved renal NRF2 protein and facilitated NRF2 nuclear translocation and function, without altering Nrf2 mRNA. To test the effect of SP600125 on Nrf2 gene expression and function, (A) Nrf2 mRNA, protein levels of (B) t-NRF2 and (C) n-NRF2 were determined in both types of the mice. In order to evaluate NRF2 nuclear translocation, (D) ratio of n-NRF2/Histione H3 to t-NRF2/GAPDH was calculated. Further, NRF2 function was evaluated by determining the mRNA expression of (E) Ho1 and (F) NqO1. IHC staining of (G) HO1 and (H) NQO1 was further performed to assess the status of renal antioxidant activity. The data are normalized to WT Ctrl and presented as means \pm s.D. $(n=8) .{ }^{*} P<0.05$ vs WT Ctrl; ${ }^{+} P<0.05$ vs WT DM. Bars: white, Ctrl; light gray, Ctrl/JNKi; dark gray, DM; black, DM/JNKi. Ho1, heme oxygenase 1; n-NRF2, nuclear NRF2; Nqo1, NAD(P)H dehydrogenase (quinone) 1; t-NRF2, total cellular NRF2. Other abbreviations are the same as those in Figs 1 and 2.

tested in HG-treated MMCs whether both SP600125 and JNK siRNA could preserve NRF2 protein and trigger its nuclear translocation. As shown in Fig. 5, both SP600125 and JNK siRNA increased t-NRF2 (Fig. 5A),
n-NRF2 (Fig. 5B) and ratio of n-NRF2/histone $\mathrm{H} 3$ to t-NRF2/GAPDH (Fig. 5C). The two JNK inhibiting approaches further enhanced the mRNA levels of Ho1 and Nqo1 (Fig. 5D and E). 
A

\begin{tabular}{l}
$\square$ Ctrl \\
\hline Ctrl/JNKi $_{\text {DM }}$ \\
DM/JNKi
\end{tabular}

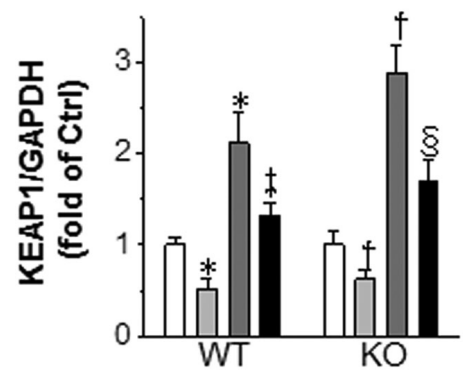

D
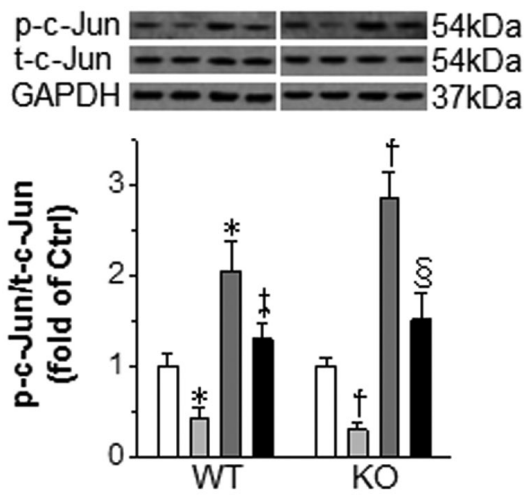

$F$
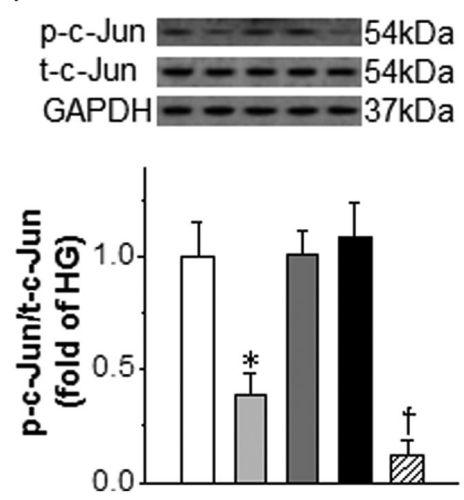

B
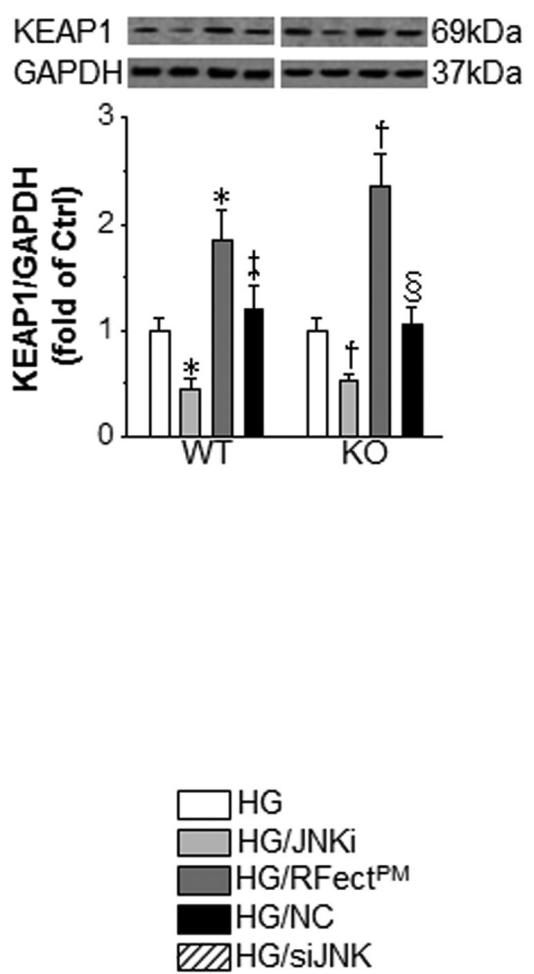

G

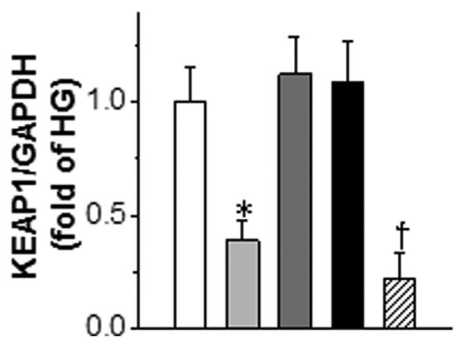

C
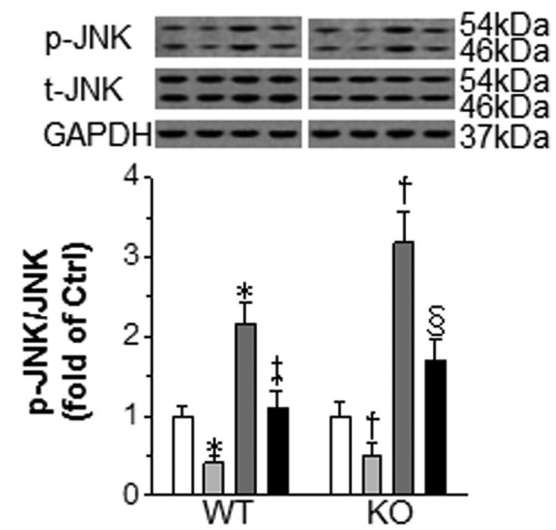

E
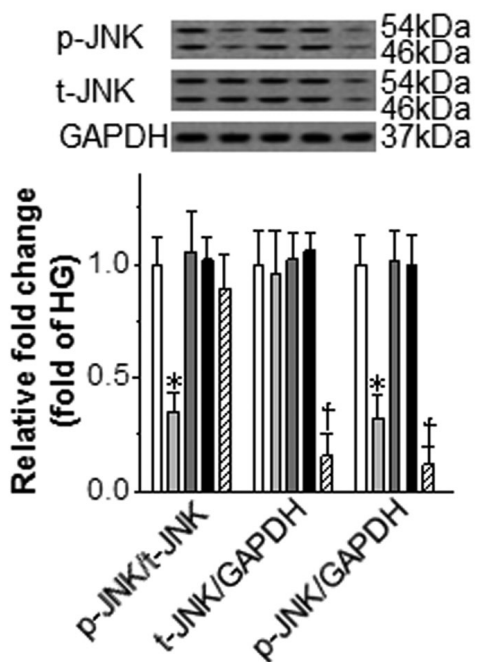

$\mathrm{H}$
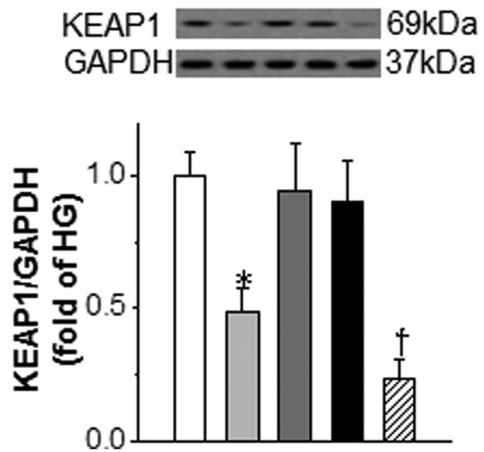

Figure 4

SP600125 decreased Keap1 expression through inhibition of JNK activity. With the aim of exploring the mechanism by which SP600125 activates NRF2, Keap1 (A) mRNA and (B) protein, as well as ratios of (C) p-JNK to t-JNK and (D) p-c-Jun to t-c-jun were determined in the kidneys of the WT and Nrf2 KO mice. To further define the effect of JNK inhibition on Keap1 expression, MMCs were treated with HG, in the presence of either SP600125 or JNK siRNA, with ratios of (E) p-JNK to t-JNK, t-JNK to GAPDH, p-JNK to GAPDH and (F) p-c-Jun to t-c-jun, as well as Keap1 (G) mRNA and (H) protein determined. For $(\mathrm{A}, \mathrm{B}, \mathrm{C}$ and $\mathrm{D})$, the data is normalized to respective Ctrls and presented as means \pm S.D. $(n=8) .{ }^{*} P<0.05$ vs WT Ctrl; ${ }^{\dagger} P<0.05$ vs $\mathrm{KO} C$ trl; ${ }^{\ddagger} P<0.05$ vs WT DM; $\S P<0.05$ vs KO DM. Bars: white, Ctrl; light gray, Ctrl/JNKi; dark gray, DM; black, DM/JNKi. For (E, F, G and H), the data are normalized to HG and presented as means \pm s.D. $(n=3)$. ${ }^{*} P<0.05$ vs HG; ${ }^{+} P<0.05$ vs HG/NC. Bars: white, HG; light gray, HG/JNKi; dark gray, HG/RFectPM; black, HG/NC; white with stripes, HG/ siJNK. HG, high glucose; Keap1, Kelch-like ECH-associated protein 1; NC, negative control siRNA; p-c-Jun, phosphorylated c-Jun; p-JNK, phosphorylated JNK; RFectPM, the transfection reagent; siJNK, JNK siRNA; t-c-Jun, total c-Jun; t-JNK, total JNK. Other abbreviations are the same as those in Fig. 1. 
A
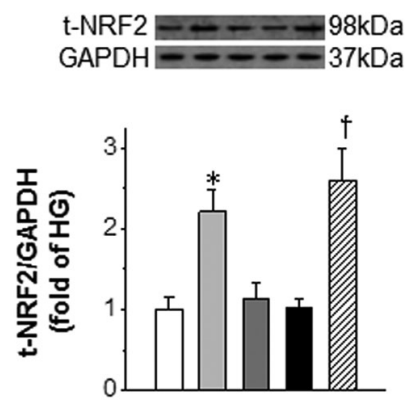

C

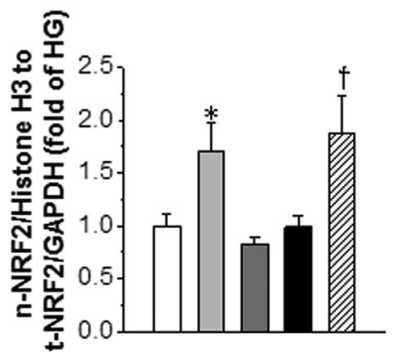

$E$

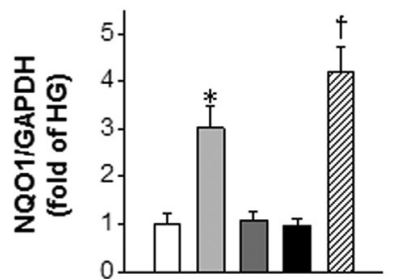

$B$

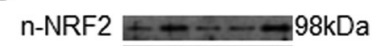

Histone $\mathrm{H} 3=--15 \mathrm{kDa}$

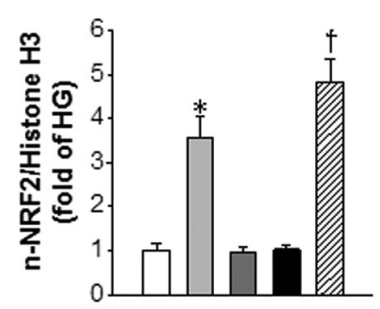

D

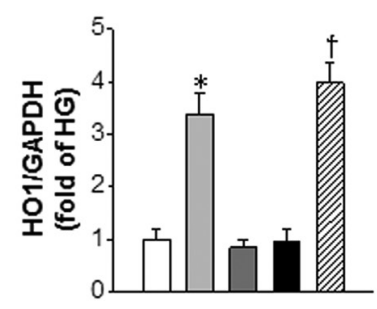

Figure 5

Both SP600125 and JNK siRNA preserved NRF2 protein and enhanced its nuclear translocation and function. In order to test the effect of JNK inhibition on NRF2 expression and function, MMCs were subjected to HG, in the presence of either SP600125 or JNK siRNA. (A) t-NRF2 and (B) n-NRF2 levels were determined by Western blot. (C) n-NRF2/Histione H3 to t-NRF2/GAPDH was calculated to reflect NRF2 nuclear translocation. mRNA levels of (D) Ho1 and (E) Nqo1 were determined by RT-PCR. The data are normalized to HG and presented as means \pm s.D. $(n=3) .{ }^{*} P<0.05$ vs HG; ${ }^{\dagger} P<0.05$ vs HG/NC. Bars: white, HG; light gray, HG/JNKi; dark gray, HG/RFectPM; black, HG/NC; white with stripes, HG/siJNK; Abbreviations are the same as those in Figs 3 and 4.

\section{JNK inhibition by either SP600125 or JNK siRNA decreased oxidative damage and expression of inflammatory and fibrotic genes in HG-treated MMCs}

Finally, comparing to the effect of JNK siRNA, the role of JNK inhibition in the protective effect of SP600126 was tested by determining MDA levels (Fig. 6A) as well as mRNA expression of $i$ Nos (Fig. 6B), Vcam-1 (Fig. 6C), Icam1 (Fig. 6D), Fn (Fig. 6E) and Col4 (Fig. 6F) in HG-treated MMCs. All these parameters were significantly decreased by SP600125 or JNK siRNA (Fig. 6A, B, C, D, E and F).

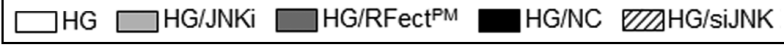

A

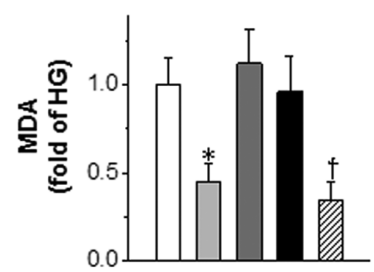

C

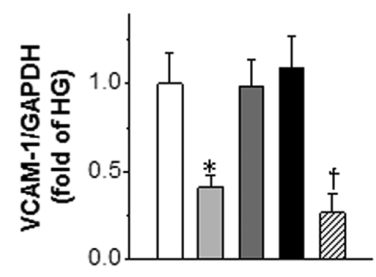

E

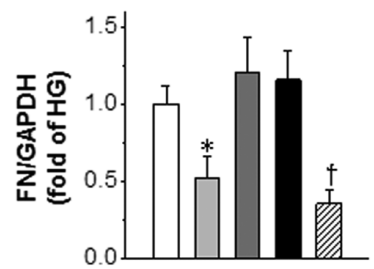

B

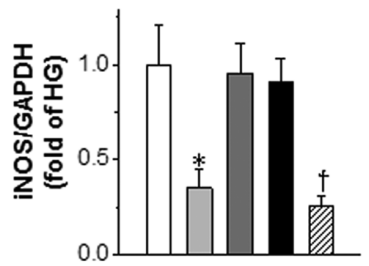

D

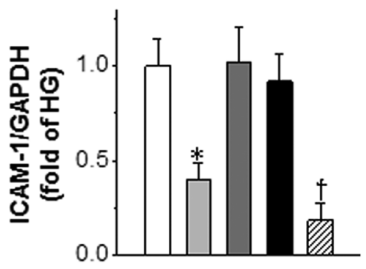

$\mathrm{F}$

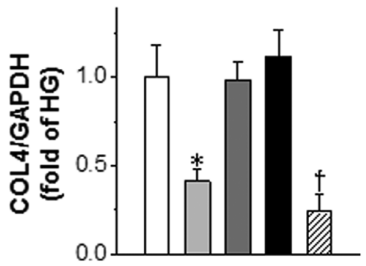

Figure 6

JNK inhibition by either SP600125 or JNK siRNA decreased oxidative damage and expression of inflammatory and fibrotic genes in HG-treated MMCs. SP600125 and JNK siRNA were further tested for their roles in alleviating HG-induced oxidative stress, inflammation and fibrosis in MMCs. (A) MDA levels and mRNA expression of (B) iNos, (C) Vcam-1, (D) $I c a m-1$, (E) $F n$ and (F) Col4 were determined. The data are normalized to HG and presented as means \pm s.D. $(n=3) .{ }^{*} P<0.05$ vs HG; ${ }^{+} P<0.05$ vs HG/NC. Bars: white, HG; light gray, HG/JNKi; dark gray, HG/RFectPM; black, $\mathrm{HG} / \mathrm{NC}$; white with stripes, HG/siJNK. Col4, collagen 4; Fn, fibronectin. Other abbreviations are the same as those in Figs 2 and 4.

Hence, the benefits of SP600125 were mediated by JNK inactivation.

\section{Discussion}

The present study researched the action of SP600125 in Nrf2 expression and function in the protection against DN. By using Nrf2 KO mice, NRF2 was found to be the major factor through which SP600125 ameliorated DN. In the kidneys of the WT mice, SP600125 inactivated JNK, inhibited Keap1 expression, preserved cellular NRF2 protein level and facilitated NRF2 nuclear translocation. By comparing to the effect of JNK siRNA, SP600125 was found to inhibit Keap1 expression, activate NRF2 
signaling and attenuate HG-induced deleterious effects in HG-treated MMCs via inactivation of JNK. The present study indicates a negative regulatory effect of JNK on NRF2 in DN possibly through JNK-induced Keap1 expression and may thus provide JNK inhibition as a feasible strategy for prevention of $\mathrm{DN}$.

By phosphorylating c-Jun and thereby activating AP-1(Davis 2000), JNK induces the transcription of Tgf- $\beta 1$ (Weigert et al. 2000) and Fn (Lan et al. 2013), both of which play key roles in the pathogenesis of DN (Schena \& Gesualdo 2005). Inhibition of JNK by SP600125 decreased Tgf- $\beta 1$ (Gao et al. 2016) and Fn (Wang et al. 2015, Gao et al. 2016) and ameliorated DN. It was therefore speculated that JNK may contribute to the development of DN by activating Tgf- $\beta 1$ and $F n$. In the present study, NRF2 was found to be another downstream target of JNK. Different from the positive impact on Tgf- $\beta 1$ and $F n$ gene transcription, JNK negatively regulated $\mathrm{Nr} f 2$ expression at the protein level, but not the mRNA level (Fig. 3A and B). NRF2 played a major role in the protection by SP600125, since most of the beneficial effects of SP600125 did not exist in the absence of NRF2 (Figs 1B, C, D, E, F; 2A, B, C, $\mathrm{D}, \mathrm{E}$ and 3E, F). Inhibition of Tgf- $\beta 1$ and $F n$ by SP600125 might also play important roles in SP600125's protection against DN. However, this hypothesis needs to be further tested by manipulating $T g f-\beta 1$ and $F n$ expression in the presence of SP600125 in experimental models of DN. One possibility might be that NRF2, TGF- $\beta 1$ and FN all play pivotal roles, with crosstalk existing among them, functioning at different steps as a circuit or a cascade. Supporting this view, higher expression of $T g f-\beta 1$ and $F n$ was found in the kidneys of the Nrf2 KO diabetic mice, as compared with the WT diabetic mice (Jiang et al. 2010, $\mathrm{Wu}$ et al. 2016). This indicates that NRF2 negatively regulates $T g f-\beta 1$ and $F n$ expression. Thus, upon diabetes, in addition to the JNK-induced AP-1 transcriptional activity, JNK might enhance Tgf- $\beta 1$ and $F n$ expression via inhibition of NRF2. The correlation between NRF2, TGF- $\beta 1$ and $\mathrm{FN}$ in $\mathrm{DN}$ needs to be further researched in future studies. In addition to the potential NRF2/TGF- $31 / \mathrm{FN}$ network, other mechanisms, such as anti-inflammation (Pan et al. 2013) and anti-renin-angiotensin system (Hong et al. 2016) may also contribute to SP600125's protection. Notably, NRF2 activation by SP600125 might also account for the anti-inflammatory function of SP600125, given that SP600125 completely lost the ability to attenuate the pro-inflammatory factors TNF- $\alpha$ and VCAM-1 (Fig. 2D and E) in the absence of NRF2. In the present study, a slight, but significant, decrease in Masson's positive area (Fig. 1G, right panel), and CTGF protein (Fig. 2G, right panel) was detected in the kidneys of the SP600125treated Nrf2 KO diabetic mice. These observations suggest a NRF2-independent mechanism, which plays a partial role in SP600125's attenuation of diabetes-induced renal fibrosis, although the outcome of this mechanism was minor compared to the potent effect of NRF2 (Figs 1B, C, D, E, F and 2A, B, C, D, E).

Small molecules have been designed to disable KEAP1. Dimethyl fumarate, bardoxolone methyl (Wang et al. 2014) and sulforaphane (SFN) (Zhang \& Hannink 2003) are the well-known small molecules that modify critical cysteines within KEAP1 protein, leading to the release of NRF2 (Takaya et al. 2012). Among these KEAP1 inhibitors, dimethyl fumarate is currently in use for clinical treatment of multiple sclerosis (Gold et al. 2012), demonstrating NRF2 as a viable drug target. Different from these KEAP1 inhibitors, SP600125 was found, in the present study, to decrease Keap1 expression through inactivation of JNK, followed by NRF2 activation. The present study may suggest inhibition of Keap1 expression as a strategy to activate NRF2, in addition to structural modification of the KEAP1 protein. In addition, the present study also suggests JNK gene silencing as an effective approach to activate NRF2 (Figs 4E, F, G, H and 5A, B, C, D, E). Both SP600125 and JNK siRNA decreased the amount of p-JNK (Fig. 4E, right panel), which reflected an inhibited JNK activity. However, SP600125 and JNK siRNA were found, in the present study, to inactivate JNK through different mechanisms. Specifically, SP600125 inactivated JNK by inhibiting its phosphorylation (Fig. 4E, left panel), whereas JNK siRNA via decreasing the amount of JNK (Fig. 4E, middle panel).

Controversies exist within establishing the effect of MAPKs on the regulation of NRF2 signaling. It was assumed that activation of MAPKs may phosphorylate NRF2 at specific serine and threonine residues, thereby facilitating the release of NRF2 from KEAP1 (Lee \& Surh 2005). However, opposed JNK activity and NRF2 expression were observed in experimental models of various diseases (Tan et al. 2013, Yenki et al. 2013, Sahu et al. 2015, Ye et al. 2015, Du et al. 2016). Additionally, our previous report showed that inhibition of JNK by SP600125 or C66 led to NRF2 activation in the aortas of the diabetic mice (Liu et al. 2014). In line with these studies, the present study demonstrated a negative impact of JNK on NRF2 expression (Figs 3B, C, D, E, F, G and 5A, B, C, D, E). Collectively, these findings shed light on diverse regulatory effects of JNK on NRF2. One possibility for the differential behaviors of JNK could be the difference between disease conditions or cell types. A specific effect of JNK may play 
a major role under a certain condition. The final outcome may be the result of a combination of all the actions of JNK. In the present study, enhancing Keap1 expression was the most predominant action of JNK in inhibiting NRF2. Although JNK-induced phosphorylation of NRF2 and adaptive response to the oxidative stress might play positive roles in activating NRF2, these possible effects could be minor, as compared with the effect of JNK on inducing Keap1 expression.

In our previous study, SP600125 increased both Nrf2 mRNA and protein in the aorta (Liu et al. 2014). However, renal Nrf2 mRNA was not altered by SP600125 in the present study (Fig. 3A, left panel). This could be due to the variation of the JNK function in different organs or cell types. Nonetheless, our previous study did show an increased nuclear positive staining of NRF2 in the aorta (Liu et al. 2014), suggesting an enhanced NRF2 nuclear translocation by SP600125. SP600125's negative effect on Keap1 expression has been further tested and verified in the present study (Fig. 4A, B, G and H). In our previous report, metallothionein (MT), a potent antioxidant, was shown to be an important downstream target of NRF2 in DN (Wu et al. 2015), since deletion of the Mt gene partially, but significantly, abolished the protective effects of the NRF2 activator SFN. The rest of the protection produced by SFN in the absence of MT might be due to the NRF2-induced activation of other canonical downstream targets, such as Ho1 and Nqo1.

It is noted that different stages of diabetic complications may have different status of antioxidant defensive function. Nrf2 expression and function were upregulated in the aorta (Liu et al. 2014), heart (Bai et al. 2013) and kidney (Cui et al. 2012) of mice 3 months post diabetes onset, which was a relatively early stage of diabetes in mice. The activation of NRF2 antioxidant system could be due to the compensatory response to the stimulation by diabetes-induced oxidative stress (Bai et al. 2013), the effect of which adaptively overcomes the diabetes-induced injury. However, after a long-term exposure to diabetes-induced oxidative damage, the NRF2 antioxidant system tends to lose this function. This is evidenced by the impaired Nrf2 expression and function in the hearts and kidneys of diabetic mice, 6 months post the onset of diabetes (Bai et al. 2013, Wu et al. 2016). In fact, decreased Nrf2 expression was also observed in the hearts of diabetic patients as a chronic complication of diabetes (Tan et al. 2011). This could possibly be caused by the impaired compensatory function of the antioxidant defensive response after a long-term exposure to hyperglycemia. In the present study, enhanced JNK function and Keap1 expression in the 6-month diabetic kidneys might account for the impaired NRF2 function. Further studies are needed to elucidate the mechanism for the impaired $\mathrm{Nrf2}$ expression and function at the late stage of diabetes. In the present study, although renal NRF2 signaling was decreased in the WT diabetic mice (Fig. 3B, $\mathrm{C}, \mathrm{D}, \mathrm{E}$ and F, left panels), the presence of the basal NRF2 did play a protective role in the prevention of $\mathrm{DN}$, since the Nrf2 KO diabetic mice developed more severe renal dysfunction (Fig. 1B) and pathological changes (Fig. 1C, D, E, F and G), as compared with the WT diabetic mice.

Taken together, the present study demonstrates, for the first time, that NRF2 plays a critical role in SP600126 prevention of DN. Other findings suggest JNK to be a negative regulator of NRF2 in DN, the effect of which may be mediated by JNK-induced Keap1 expression. This study may provide a basis for understanding the effect of JNK in DN and may indicate JNK inactivation, JNK gene silencing and inhibition of Keap1 expression as potential strategies for prevention of DN.

Declaration of interest

The authors declare that there is no conflict of interest that could be perceived as prejudicing the impartiality of the research reported.

\section{Funding}

This work was supported in part by the National Natural Science Foundation of China (81600573 to H W), Norman Bethune Program of Jilin University (2015438 to H W), Department of Science and Technology of Jilin Province (20170204032YY to J W), Development and Reform Commission of Jilin Province (2014G072 to J W) and Research Program of Jilin University (3R2173203428 to Y J).

\section{Author contribution statement}

$\mathrm{H} \mathrm{W}$ conceived the project. $\mathrm{H} \mathrm{W}, \mathrm{J} \mathrm{W}$ and $\mathrm{H} \mathrm{Z}$ designed the research. $\mathrm{H} \mathrm{Z}$, $X \mathrm{~L}, \mathrm{~S} Z, Y \mathrm{~J}, \mathrm{Y} \mathrm{L}$ and $\mathrm{Y} \mathrm{S}$ researched the data. $\mathrm{H} \mathrm{W}, \mathrm{J} W$ and $\mathrm{H} Z$ wrote the manuscript. J W, H W, Y J, H Z, X L, S Z, Y L and Y S stimulated discussion. $H Z, X L, S Z, Y J, Y L, Y S, J W$ and $H W$ reviewed and revised the manuscript. $\mathrm{H} \mathrm{W}, \mathrm{J} \mathrm{W}$ and $\mathrm{Y} J$ provided funding. All the authors approved the version to be published.

\section{References}

Bai Y, Cui W, Xin Y, Miao X, Barati MT, Zhang C, Chen Q, Tan Y, Cui T, Zheng Y, et al. 2013 Prevention by sulforaphane of diabetic cardiomyopathy is associated with up-regulation of Nrf2 expression and transcription activation. Journal of Molecular and Cellular Cardiology 57 82-95. (https://doi.org/10.1016/j.yjmcc.2013.01.008) Bogoyevitch MA, Boehm I, Oakley A, Ketterman AJ \& Barr RK 2004 Targeting the JNK MAPK cascade for inhibition: basic science and therapeutic potential. Biochimica et Biophysica Acta 1697 89-101. (https://doi.org/10.1016/j.bbapap.2003.11.016) 
Cai L, Wang J, Li Y, Sun X, Wang L, Zhou Z \& Kang YJ 2005 Inhibition of superoxide generation and associated nitrosative damage is involved in metallothionein prevention of diabetic cardiomyopathy. Diabetes 54 1829-1837. (https://doi.org/10.2337/diabetes.54.6.1829)

Cui W, Bai Y, Miao X, Luo P, Chen Q, Tan Y, Rane MJ, Miao L \& Cai L 2012 Prevention of diabetic nephropathy by sulforaphane: possible role of Nrf2 upregulation and activation. Oxidative Medicine and Cellular Longevity 2012 821936. (https:doi.org/10.1155/2012/821936)

Davis RJ 2000 Signal transduction by the JNK group of MAP kinases. Cell 103 239-252. (https://doi.org/10.1016/S0092-8674(00)00116-1)

De Borst MH, Prakash J, Melenhorst WB, van den Heuvel MC, Kok RJ, Navis G \& van Goor H 2007 Glomerular and tubular induction of the transcription factor c-Jun in human renal disease. Journal of Pathology 213 219-228. (https://doi.org/10.1002/path.2228)

Dong W, Jia Y, Liu X, Zhang H, Li T, Huang W, Chen X, Wang F, Sun W \& Wu H 2017 Sodium butyrate activates NRF2 to ameliorate diabetic nephropathy possibly via inhibition of HDAC. Journal of Endocrinology 232 71-83. (https://doi.org/10.1530/JOE-16-0322)

Dronavalli S, Duka I \& Bakris GL 2008 The pathogenesis of diabetic nephropathy. Nature Clinical Practice Endocrinology and Metabolism 4 444-452. (https://doi.org/10.1038/ncpendmet0894)

Du J, Zhang M, Lu J, Zhang X, Xiong Q, Xu Y, Bao Y \& Jia W 2016 Osteocalcin improves nonalcoholic fatty liver disease in mice through activation of Nrf2 and inhibition of JNK. Endocrine $\mathbf{5 3}$ 701-709. (https://doi.org/10.1007/s12020-016-0926-5)

Gao P, Wei Y, Zhang Z, Zeng W, Sun D, Liu D, Hou B, Zhang C, Zhang N, Li H, et al. 2016 Synergistic effects of c-Jun and SP1 in the promotion of TGFbeta1-mediated diabetic nephropathy progression. Experimental and Molecular Pathology 100 441-450. (https://doi. org/10.1016/j.yexmp.2016.04.005)

Gold R, Kappos L, Arnold DL, Bar-Or A, Giovannoni G, Selmaj K, Tornatore C, Sweetser MT, Yang M, Sheikh SI, et al. 2012 Placebocontrolled phase 3 study of oral BG-12 for relapsing multiple sclerosis. New England Journal of Medicine 367 1098-1107. (https:// doi.org/10.1056/NEJMoa1114287)

Ho C, Lee PH, Huang WJ, Hsu YC, Lin CL \& Wang JY 2007 Methylglyoxal-induced fibronectin gene expression through Rasmediated NADPH oxidase activation in renal mesangial cells. Nephrology 12 348-356. (https://doi. org/10.1111/j.1440-1797.2007.00809.x)

Hong Z, Hong Z, Wu D \& Nie H 2016 Specific MAPK inhibitors prevent hyperglycemia-induced renal diseases in type 1 diabetic mouse model. Molecular and Cellular Biochemistry 419 1-9. (https://doi. org/10.1007/s11010-016-2722-1)

Jiang T, Huang Z, Lin Y, Zhang Z, Fang D \& Zhang DD 2010 The protective role of Nrf2 in streptozotocin-induced diabetic nephropathy. Diabetes 59 850-860. (https://doi.org/10.2337/db091342)

Kensler TW, Wakabayashi N \& Biswal S 2007 Cell survival responses to environmental stresses via the Keap1-Nrf2-ARE pathway. Annual Review of Pharmacology and Toxicology 47 89-116. (https://doi. org/10.1146/annurev.pharmtox.46.120604.141046)

Lan T, Wu T, Gou H, Zhang Q, Li J, Qi C, He X, Wu P \& Wang L 2013 Andrographolide suppresses high glucose-induced fibronectin expression in mesangial cells via inhibiting the AP-1 pathway. Journal of Cellular Biochemistry 114 2562-2568. (https://doi. org/10.1002/jcb.24601)

Lee JS \& Surh YJ 2005 Nrf2 as a novel molecular target for chemoprevention. Cancer Letters 224 171-184. (https://doi. org/10.1016/j.canlet.2004.09.042)

Liu Y, Wang Y, Miao X, Zhou S, Tan Y, Liang G, Zheng Y, Liu Q, Sun J \& Cai L 2014 Inhibition of JNK by compound C66 prevents pathological changes of the aorta in STZ-induced diabetes. Journal of Cellular and Molecular Medicine 18 1203-1212. (https://doi. org/10.1111/jcmm.12267)
Pan Y, Zhang X, Wang Y, Cai L, Ren L, Tang L, Wang J, Zhao Y, Wang Y, Liu Q, et al. 2013 Targeting JNK by a new curcumin analog to inhibit NF-kB-mediated expression of cell adhesion molecules attenuates renal macrophage infiltration and injury in diabetic mice. PLOS ONE 8 e79084. (https://doi.org/10.1371/journal.pone.0079084)

Pan Y, Wang Y, Zhao Y, Peng K, Li W, Wang Y, Zhang J, Zhou S, Liu Q, Li X, et al. 2014 Inhibition of JNK phosphorylation by a novel curcumin analog prevents high glucose-induced inflammation and apoptosis in cardiomyocytes and the development of diabetic cardiomyopathy. Diabetes 63 3497-3511. (https://doi.org/10.2337/ db13-1577)

Ruiz S, Pergola PE, Zager RA \& Vaziri ND 2013 Targeting the transcription factor Nrf2 to ameliorate oxidative stress and inflammation in chronic kidney disease. Kidney International $\mathbf{8 3}$ 1029-1041. (https://doi.org/10.1038/ki.2012.439)

Sahu BD, Mahesh Kumar J \& Sistla R 2015 Baicalein, a bioflavonoid, prevents cisplatin-induced acute kidney injury by up-regulating antioxidant defenses and down-regulating the MAPKs and NF-kappaB pathways. PLoS ONE 10 e0134139. (https://doi. org/10.1371/journal.pone.0134139)

Schena FP \& Gesualdo L 2005 Pathogenetic mechanisms of diabetic nephropathy. Journal of the American Society of Nephrology $\mathbf{1 6}$ (Supplement 1) S30-S33. (https://doi.org/10.1681/ASN.2004110970)

Sun W, Liu X, Zhang H, Song Y, Li T, Liu X, Liu Y, Guo L, Wang F, Yang T, et al. 2017 Epigallocatechin gallate upregulates NRF2 to prevent diabetic nephropathy via disabling KEAP1. Free Radical Biology and Medicine 108 840-857. (https://doi.org/10.1016/j. freeradbiomed.2017.04.365)

Takaya K, Suzuki T, Motohashi H, Onodera K, Satomi S, Kensler TW \& Yamamoto M 2012 Validation of the multiple sensor mechanism of the Keap1-Nrf2 system. Free Radical Biology and Medicine 53 817-827. (https://doi.org/10.1016/j.freeradbiomed.2012.06.023)

Tan Y, Ichikawa T, Li J, Si Q, Yang H, Chen X, Goldblatt CS, Meyer CJ, Li X, Cai L, et al. 2011 Diabetic downregulation of Nrf2 activity via ERK contributes to oxidative stress-induced insulin resistance in cardiac cells in vitro and in vivo. Diabetic 60 625-633. (https://doi. org/10.2337/db10-1164)

Tan M, Ouyang Y, Jin M, Chen M, Liu P, Chao X, Chen Z, Chen X, Ramassamy C, Gao Y, et al. 2013 Downregulation of Nrf2/HO-1 pathway and activation of JNK/c-Jun pathway are involved in Homocysteic acid-induced cytotoxicity in HT-22 cells. Toxicology Letters 223 1-8. (https://doi.org/10.1016/j.toxlet.2013.08.011)

Wang Y, Feng W, Xue W, Tan Y, Hein DW, Li XK \& Cai L 2009 Inactivation of GSK-3beta by metallothionein prevents diabetesrelated changes in cardiac energy metabolism, inflammation, nitrosative damage, and remodeling. Diabetes 58 1391-1402. (https://doi.org/10.2337/db08-1697)

Wang YY, Yang YX, Zhe H, He ZX \& Zhou SF 2014 Bardoxolone methyl (CDDO-Me) as a therapeutic agent: an update on its pharmacokinetic and pharmacodynamic properties. Drug Design, Development and Therapy 8 2075-2088. (https://doi.org/10.2147/ DDDT.S68872)

Wang Y, Wang Y, Luo M, Wu H, Kong L, Xin Y, Cui W, Zhao Y, Wang J, Liang G, et al. 2015 Novel curcumin analog C66 prevents diabetic nephropathy via JNK pathway with the involvement of p300/CBPmediated histone acetylation. Biochimica et Biophysica Acta 1852 34-46. (https://doi.org/10.1016/j.bbadis.2014.11.006)

Weigert C, Sauer U, Brodbeck K, Pfeiffer A, Haring HU \& Schleicher ED 2000 AP-1 proteins mediate hyperglycemia-induced activation of the human TGF-beta1 promoter in mesangial cells. Journal of the American Society of Nephrology 11 2007-2016.

Wu H, Zhou S, Kong L, Chen J, Feng W, Cai J, Miao L \& Tan Y 2014 Metallothionein deletion exacerbates intermittent hypoxia-induced renal injury in mice. Toxicology Letters 232 340-348. (https://doi. org/10.1016/j.toxlet.2014.11.015) 
Wu H, Kong L, Cheng Y, Zhang Z, Wang Y, Luo M, Tan Y, Chen X, Miao L \& Cai L 2015 Metallothionein plays a prominent role in the prevention of diabetic nephropathy by sulforaphane via up-regulation of Nrf2. Free Radical Biology and Medicine 89 431-442. (https://doi.org/10.1016/j.freeradbiomed.2015.08.009)

Wu H, Kong L, Tan Y, Epstein PN, Zeng J, Gu J, Liang G, Kong M, Chen X, Miao L, et al. 2016 C66 ameliorates diabetic nephropathy in mice by both upregulating NRF2 function via increase in miR200a and inhibiting miR-21. Diabetologia 59 1558-1568. (https://doi. org/10.1007/s00125-016-3958-8)

Ye T, Zhen J, Du Y, Zhou JK, Peng A, Vaziri ND, Mohan C, Xu Y \& Zhou XJ 2015 Green tea polyphenol (-)-epigallocatechin-3-gallate restores Nrf2 activity and ameliorates crescentic glomerulonephritis. PLOS ONE 10 e0119543. (https://doi.org/10.1371/journal.pone.0119543)

Yenki P, Khodagholi F \& Shaerzadeh F 2013 Inhibition of phosphorylation of JNK suppresses Abeta-induced ER stress and upregulates prosurvival mitochondrial proteins in rat hippocampus. Journal of Molecular Neuroscience 49 262-269. (https://doi. org/10.1007/s12031-012-9837-y)

Zhang DD \& Hannink M 2003 Distinct cysteine residues in Keap1 are required for Keap1-dependent ubiquitination of Nrf2 and for stabilization of Nrf2 by chemopreventive agents and oxidative stress. Molecular and Cellular Biology 23 8137-8151. (https://doi. org/10.1128/MCB.23.22.8137-8151.2003)

Zheng H, Whitman SA, Wu W, Wondrak GT, Wong PK, Fang D \& Zhang DD 2011 Therapeutic potential of Nrf2 activators in streptozotocin-induced diabetic nephropathy. Diabetes $\mathbf{6 0}$ 3055-3066. (https://doi.org/10.2337/db11-0807)

Zhou X, Feng Y, Zhan Z \& Chen J 2014 Hydrogen sulfide alleviates diabetic nephropathy in a streptozotocin-induced diabetic rat model. Journal of Biological Chemistry 289 28827-28834. (https://doi. org/10.1074/jbc.M114.596593)

Received in final form 11 December 2017

Accepted 22 December 2017

Accepted Preprint published online 22 December 2017 